\title{
Analysis of the Measurement Matrix in Directional Predictive Coding for Compressive Sensing of Medical Images
}

\author{
G.Kowsalya* and A. Hepzibah Christinal* and D. Abraham Chandy ${ }^{+}$and \\ S. Jebasingh* and Chandrajit L. Bajaj ${ }^{@}$ \\ * Department of Mathematics, Karunya Institute of Technology and Sciences, Coimbatore, India. \\ ${ }^{+}$Department of Electronics and Communication Engineering, Karunya Institute of Technology and Sciences, Coimbatore, India. \\ ${ }^{\circledR}$ Computational Applied Mathematics Chair in Visualization, Institute for Computational Engineering and Sciences, University of Texas, Austin \\ Received 16 March 2021; Accepted 26 December 2021
}

\begin{abstract}
Compressive sensing of 2D signals involves three fundamental steps: sparse representation, linear measurement matrix and recovery of the signal. This paper focuses on analysing the efficiency of various measurement matrices for compressive sensing of medical images based on theoretical predictive coding. During encoding, the prediction is efficiently chosen by four directional predictive modes for block based compressive sensing measurements. In this work, Gaussian, Bernoulli, Laplace, Logistic and Cauchy random matrices are used as the measurement matrices. While decoding, the same optimal prediction is dequantized. Peak-signal-to-noise ratio and sparsity is used for evaluating the performance of measurement matrices. Experimental result shows that the spatially directional predictive coding (SDPC) with Laplace measurement matrices performs better compared to scalar quantization (SQ) and differential pulse code modulation (DPCM) methods. The results indicate that Laplace measurement matrix is the most suitable in compressive sensing of medical images.
\end{abstract}

Key Words: compressive sensing, predictive coding, measurement matrix, medical images.

\section{Introduction}

Compressive sensing (CS) is a path-breaking development in signal processing. Compressive sensing involves signal acquisition and compression mechanism and thereby the signal is captured in its compressed form. Compressive sensing is not sensing and compression but compressing while sensing itself. The compressive sensing has three stages of processing namely, sparse representation, linear measurement and recovery or reconstruction of the image. It requires the images to be sparse, which is valid as most of the medical images are sparse. Based on the literature, the challenges in compressive sensing are computationally expensive reconstruction process and dimension reduction [12].Compressive sensing is applied in many fields, such as Image processing [18], [20], signal processing [11], medical imaging [19], spectral and hyper-spectral imaging [22], Radar imaging [22] and sampling theory [11]. Generally in medical imaging the compressive sensing has been used

Correspondence to: christyhep@gmail.com

Recommended for acceptance by Angel D. Sappa

https://doi.org/10.5565/rev/elcvia.1412, ELCVIA ISSN:1577-5097

Published by Computer Vision Center / Universitat Autònoma de Barcelona, Barcelona, Spain 
to obtain a high resolution image with low noise. Recently to address the challenges, many algorithms have been developed by using block based compressive sampling [3]. Sparse representation, linear measurement and Recovery are the steps where regular developments are happening. The sparse process is implemented by projecting an original image on a suitable basis where it is represented as sparse. If an image is sparse then the matrix representation of the image contains most of the elements as zero [6]. For examples, some of the projection basis used in compressive sensing is the Contourlet transform, Fast Fourier transform (FFT), discrete wavelet transform (DWT), Dual-tree transform (DDWT) and Discrete cosine transform. In case of signal being sparse in original domain, the sparse representation process can be ignored. Otherwise it is taken care by means of mathematical transforms. In the CS process the difficulty is in selecting a suitable measurement matrix, which may if therefore the efficient recovery of an image. In order to ensure the best recovery of the image, the measurement matrix must satisfy Restricted Isometric Property (RIP).

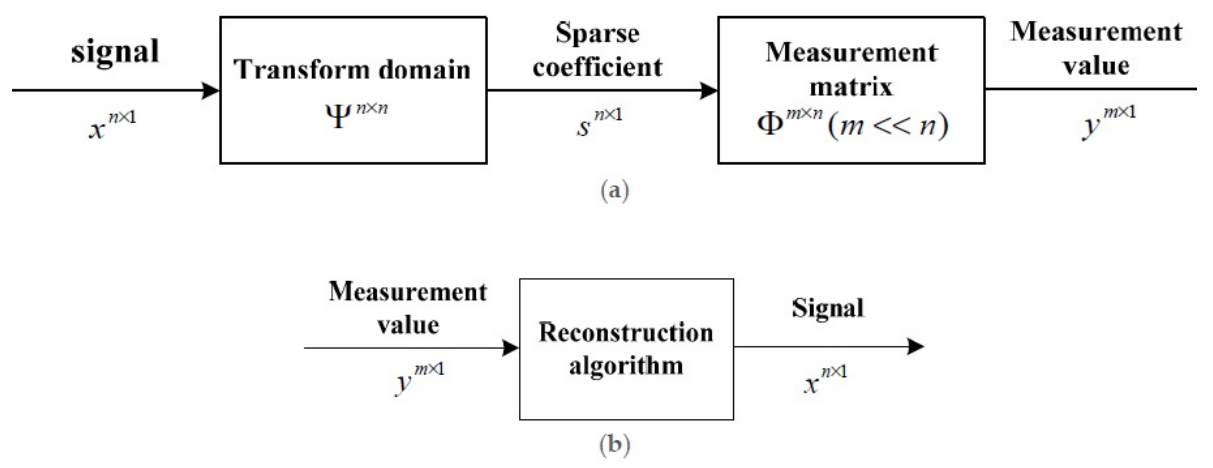

Figure 1: Compressive sensing of the signal

Many algorithms have been developed based on CS theory. Duarte et al [7] developed a single-pixel compressive sensing camera which is based on the concept of CS theory. James E. flower et al proposed the scalar quantization with differential pulse based modulation which is called the block based compressive sensing (BCS) [4]. Jian zhang et al proposed the spatially directional predictive coding with Gaussian random matrix as the measurement matrix for natural images [15].

The objective of this work is to analyse the effectiveness of various measurement matrices for directional predictive coding of medical images. This involves the comparison of SQ, DPCM, SDPC of the compressed images followed by reconstruction using Gaussian, Bernoulli, Laplace, Logistic and Cauchy measurement matrices is to have More than $30 \mathrm{~dB}$ Peak Signal to Noise Ratio (PSNR) criterion for the selection of measurement matrix for the reconstruction of image. This paper is organized as follows: in Section 2 overview of the predictive coding is discussed. In Section 3 Laplace, Logistic and Cauchy measurement matrices satisfies RIP is demonstrated using Mat-lab. Experimental results are shown in Section 4 and conclusions are drawn in Section 5 .

\section{Overview of the Predictive Coding}

In compressive sensing, sparsity is one of the important fundamental processes. If a signal is sparse in original domain or in some transformed domain by extension then CS allows exact recovery of the signal from its time or space measurements acquired by linear projection. The sparse image can be compressed to low dimensional image using the measurement matrix [3]. Subsequently the original image is restored using measurement matrix and reconstruction algorithm. The detail of the predictive coding is given below.

The input image $\mathrm{x}$ is divided into $\mathrm{n}$ non-overlapped blocks of size $B \times B$ in vector representation along the horizontal scan order, each block is denoted by $x^{i} \in R^{B^{2}}, \quad i=1,2, \ldots, n$ Then all the blocks of CS 




Figure 2: Block diagram for spatially directional predictive coding

measurements denoted by

$$
y^{i}=\phi_{B}\left(x^{i}\right)
$$

are estimated, where $y^{i} \in R^{M_{B}}$ and $\phi_{B}$ are $M_{B} \times B^{2}$ orthonormal measurement matrices with $M_{B}=\frac{M}{N} B^{2}$ . The usual choice for the measurement basis $\phi$ is a random matrix. Here, the choices are Gaussian, Bernoulli, Laplace, Logistic and Cauchy random matrices. The encoder section can design four directional prediction modes from its neighbouring reconstructed measurements namely vertical, horizontal, approximation and diagonal. More specially, let $y_{A}^{i}, y_{B}^{i}$ and $y_{C}^{i}$ denote the up-left, up, and left blocks respectively of measurements with regard to $y^{i}$. The four modes are represented as,

$$
\begin{array}{cc}
\text { Vertical mode } & y_{V}^{i}=y_{B}^{i} \\
\text { Horizontal mode } & y_{H}^{i}=y_{C}^{i} \\
\text { Approximation mode } & y_{D C}^{i}=\left(y_{B}^{i}+y_{C}^{i}\right)>>1 \\
\text { Diagonal mode } & y_{D i a g}^{i}=y_{A}^{i}
\end{array}
$$

where $>>$ the symbol denotes the right shift operator. The collection of four prediction results defined in a set $\Xi$,

$$
\Xi=\left\{y_{V}^{i}, y_{H}^{i}, y_{D C}^{i}, y_{D i a g}^{i}\right\}
$$

The optimal prediction denoted by $y_{P}^{i}$, for the measurements of the current block is then determined by minimizing the residue between $y^{i}$ and the measurement of four predictive results in set $\Xi$.

$$
y_{P}^{i}=\operatorname{argmin}_{y \in \Xi}\left\|y-y^{i}\right\|_{l_{1}}
$$

Here, $\|*\|_{l_{1}}$ is $l_{1}$ the norm, adding all the absolute values of the entries in a vector. After obtaining the optimal prediction value of $y^{i}$, the residual can be calculated by

$$
d^{(i)}=y^{i}-y_{P}^{i}
$$

Which is then scalar quantized to acquire the quantization index,

$$
s^{(i)}=Q\left[d^{(i)}\right]
$$

The operation of de-quantization of $s^{(i)}$ is then conducted to get the quantized residual $d^{i}$ which is then added by $y_{P}^{i}$, producing the reconstructed CS measurements of $y^{i}$ denoted by $y^{i}$ is ready for the further prediction coding. Bit stream is composed of two parts namely the flag of best predictive mode ( 2 bit) and 
the bits to encode $s^{(i)}$ by entropy coder. It can be noted that the 2 bit overhead is almost neglected for each block when compared with exciting gains. For instance, if block size is set to be $16 \times 16$, then the overhead is only $2 / 256=0.0078 b p p[8]$. Every blocks of CS measurement use this process for achieving final bit stream. Similarly, every block in reconstructed measurement is attained from bit stream in decoder side. By algorithms of CS recovery it is then used for ultimate image reconstruction.

\section{Restricted Isometry Property of Measurement Matrices}

In compressive sensing construction of measurement matrix plays an important role. Not every matrix is suitable for compressive sensing problem. Measurement matrices satisfying RIP can be used to recover the compressed sensed medical images. The matrix which compressively senses the signal should have several properties to keep its information content for recovery after being sensed. Donoho et al [21] proposed that a measurement matrix should satisfy the following conditions:

(i) The column vector of measurement matrix must possess certain linear independence.

(ii) The column vectors are random and independent.

(iii) The solution that satisfies the sparsity is the vector that makes the norm minimum.

Candes et al proved that the restricted isometric property is the necessary condition for a measurement matrix to recover the image without distortion [1]. The characteristic of measurement matrix is to have fewer amounts of data supporting hardware implementation, possibility of algorithm optimization and broad applicability [9]. Let $\mathrm{A}$ be a matrix that satisfies the restricted isometric property (RIP) of order $\mathrm{s}$ if there exists a such that $\delta_{s} \in(0,1)$

$$
\left(1-\delta_{s}\right)\|x\|_{2}^{2} \leq\|A x\|_{2}^{2} \leq\left(1+\delta_{s}\right)\|x\|_{2}^{2}
$$

where, $x$ is the input matrix. Moreover, the matrix must satisfy the restricted Isometric property, i.e., $\left(s, \delta_{s}\right)$ with restricted constant $\delta_{s}, \delta>0$ this constant is also referred as restricted isometry constant [9]. Sparsity basis and measurement basis vectors that are orthogonal are mutually independent and have no correlation with each other. Therefore, to accomplish the pairwise independent between the vectors the measurement matrix is orthogonalized, which is possible in CS.

\subsection{RIP property of Gaussian and Bernoulli matrix:}

Let $\phi$ be the Gaussian random matrix and its elements are independent and normally distributed with expectation between 0 and 1 , mean 0 and variance $1 / \sigma$. The probability density function of a normal distribution is:

$$
f(x)=\frac{1}{\sigma \sqrt{2 \pi}} e^{\frac{-(x-\mu)^{2}}{2 \sigma^{2}}}
$$

Where $\mu$ is the mean of the distribution and $\sigma^{2}$ is the standard deviation. In general a matrix $\phi \in R^{M \times N}(M<<$ $N)$ satisfies the RIP of order $k$ if there exists a $\delta \in(0,1)$ such that,

$$
1-\delta \leq \frac{\left\|\phi x_{1}-\phi x_{2}\right\|_{2}^{2}}{\left\|x_{1}-x_{2}\right\|_{2}^{2}} \leq 1+\delta
$$

holds for all $K$-sparse vectors $x_{1}$ and $x_{2}$. Here, $M=O(k \log N)$

Let $B \in R^{M \times N}$ random Bernoulli matrix and the values of the elements are $+1 / \sqrt{M}$ and $-1 / \sqrt{M}$ with equal probabilities. The expected output will be $n=0$ and $n=1$ with equal probabilities of $p=1 / 2$ and $q=1-p=1 / 2$ respectively. Thus, the probability density function is: 


$$
f(n)=\left\{\begin{array}{lll}
\frac{1}{2} & \text { for } & n=0 \\
\frac{1}{2} & \text { for } & n=1
\end{array}\right.
$$

The RIP condition is satisfied with the probability which is same as the Random Gaussian matrix [4].

\subsection{RIP property of Random Laplace, Logistic and Cauchy matrix}

Let $\phi$ be the Laplace random matrix with mean 0 and variance 1 . The probability density function of a Laplace distribution is,

$$
f(x)=\frac{1}{2 s} e^{-|x-\mu| / s}
$$

where, $\mu$ - mean and $\mathrm{s}-$ scale parameter [17]. Let $\phi$ be the logistic random matrix and its elements are independent and identically distributed with mean 0 and variance 1 . The probability density function of a Logistic distribution is:

$$
f(x)=\frac{1}{4 x} \operatorname{sech}^{2}\left(-\frac{x-\mu}{2 s}\right) \quad-\infty<x<\infty
$$

where, $\mu$ is the mean and $\mathrm{s}$ is the scale parameter [17]. Let $\phi$ be the Cauchy random matrix and elements are random variable with mean 0 and variance 1 . The probability distribution of a Cauchy distribution is,

$$
f(x)=\frac{1}{\pi}\left[\frac{s}{(x-l)^{2}+s^{2}}\right]
$$

Where, $l$ is location parameter and $s$ is scale parameter [17].



(a) Shoulder MRI

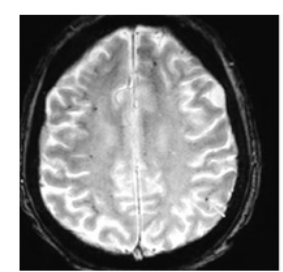

(b) Brain MRI



(c) Chest-CT

Figure 3: Sample Medical Images

\section{Experimental Results and Discussion}

In this paper, the medical images namely Shoulder MRI, Chest radiograph [23] and Brain MRI [24] as shown in Fig. 3 are considered for experimentation, where the last two types are of covid-19 patients. The details of the image data used in the experimentation are as follows: magnetic resonance image (MRI) of shoulder rotator cut tear generated in Melbourne radiology clinic; brain MRI of 61 year old male COVID-19 patient at New York university Langone medical centre; chest CT image of 59 year old female COVID-19 patient at University of Hong Kong-Shenzhen Hospital, Hong Kong West. To facilitate the computation these images are uniformly resized to $256 \times 256$ pixels of resolution $150 \mathrm{dpi}$. The software implementation of the methodology is carried out with MATLAB 2019a using an Intel Core i5 64-bit processor with 8.00 GB RAM, 2.60 GHz clock speed on the Windows 10 operating system. The measurement matrices such as Gaussian random matrix, Bernoulli random matrix, Laplace random matrix, Logistic random matrix and Cauchy random matrix are applied and reconstructed by the predictive coding techniques such as scalar quantization (SQ), differential 
pulse code modulation (DPCM) and Spatially directional predictive coding (SDPC). The metrics Mean Square Error (MSE) and PSNR are used to compare the compressive sensing results.

The formula for finding MSE and PSNR is given below:

$$
M S E=\frac{1}{N^{2}} \sum_{i, j=0}^{n-1}\|x(i, j)-y(i, j)\|^{2}
$$

where, $x(i, j)$ and $y(i, j)$ are original and reconstructed images.

$$
P S N R=10 \log \frac{\left(2^{n}-1\right)^{2}}{M S E}
$$

where, $\mathrm{n}$ is the number of bits used to represent the pixels.

$$
\text { Sparcity }=\frac{\text { no. of elements near to zero }}{\text { Total number of elements }} \times 100
$$

It is to be noted that for better image reconstruction the minimum PSNR to be obtained is $30 \mathrm{~dB}$.

Table 1: Performance Evaluation of Brain MRI

\begin{tabular}{|l|c|c|c|c|c|c|c|c|c|}
\hline $\begin{array}{l}\text { Measurement } \\
\text { Matrix Distribution }\end{array}$ & \multicolumn{3}{|c|}{ SQ } & \multicolumn{3}{c|}{ DPCM-Plus-SQ } & \multicolumn{3}{c|}{ SDPC-Plus-SQ } \\
\cline { 2 - 10 } & $\begin{array}{c}\text { PSNR } \\
(\mathrm{dB})\end{array}$ & $\begin{array}{c}\text { Time } \\
(\mathrm{sec})\end{array}$ & $\begin{array}{c}\text { Sparcity } \\
(\%)\end{array}$ & $\begin{array}{c}\text { PSNR } \\
(\mathrm{dB})\end{array}$ & $\begin{array}{c}\text { Time } \\
(\mathrm{sec})\end{array}$ & $\begin{array}{c}\text { Sparcity } \\
(\%)\end{array}$ & $\begin{array}{c}\text { PSNR } \\
(\mathrm{dB})\end{array}$ & $\begin{array}{c}\text { Time } \\
(\mathrm{sec})\end{array}$ & $\begin{array}{c}\text { Sparcity } \\
(\%)\end{array}$ \\
\hline $\begin{array}{l}\text { Gaussian } \\
\text { Random } \\
\text { Matrix }\end{array}$ & 24.08 & 8.58 & 66.36 & 34.55 & 2.43 & 57.35 & 33.87 & 1.67 & 54.67 \\
\hline $\begin{array}{l}\text { Bernoulli } \\
\text { Random } \\
\text { Matrix }\end{array}$ & 18.38 & 8.61 & 99.06 & 28.56 & 1.24 & 77.73 & 28.02 & 1.38 & 54.88 \\
\hline $\begin{array}{l}\text { Laplace } \\
\text { Random } \\
\text { Matrix }\end{array}$ & 23.27 & 8.93 & 76.58 & 34.38 & 1.79 & 56.60 & 34.83 & 2.01 & 49.13 \\
\hline $\begin{array}{l}\text { Logistic } \\
\text { Random } \\
\text { Matrix }\end{array}$ & 23.75 & 10.93 & 72.05 & 33.46 & 1.82 & 51.66 & 34.39 & 1.72 & 49.71 \\
\hline $\begin{array}{l}\text { Cauchy } \\
\text { Random } \\
\text { Matrix }\end{array}$ & 21.47 & 13.29 & 60.01 & 34.46 & 2.07 & 55.85 & 34.05 & 2.12 & 57.62 \\
\hline
\end{tabular}

In Table 1, the compressive sensing results for brain MRI dataset with reference to different measurement matrices applied in the predictive coding approaches are presented. For SQ method, the Gaussian random matrix has yielded maximum PSNR value $(24.08 \mathrm{~dB})$ and minimum computation time $(8.58 \mathrm{sec}$.$) . For the$ same, Bernoulli matrix has shown least performance in terms of PSNR and sparsity (99.06\%), whereas Cauchy random matrix requires more computation time. For DPCM-Plus-SQ method, maximum PSNR value (34.55 $\mathrm{dB})$ is attained by Gaussian random matrix, least computation time $(2.43 \mathrm{sec}$.) and sparsity $(77.73 \%)$. For SDPC-Plus-SQ method, better PSNR value $(34.83 \mathrm{~dB})$ and sparsity $(49.13 \%)$ is attained for Laplace random matrix and less computation time for Bernoulli random matrix. The least performance is for Bernoulli in terms of PSNR and Cauchy random matrix in terms of computation time and sparsity. On the whole, Laplace random 
Table 2: Performance Evaluation of Chest-CT

\begin{tabular}{|l|c|c|c|c|c|c|c|c|c|}
\hline $\begin{array}{l}\text { Measurement } \\
\text { Matrix Distribution }\end{array}$ & \multicolumn{3}{|c|}{ SQ } & \multicolumn{3}{c|}{ DPCM-Plus-SQ } & \multicolumn{3}{c|}{ SDPC-Plus-SQ } \\
\cline { 2 - 10 } & $\begin{array}{c}\text { PSNR } \\
(\mathrm{dB})\end{array}$ & $\begin{array}{c}\text { Time } \\
(\mathrm{sec})\end{array}$ & $\begin{array}{c}\text { Sparcity } \\
(\%)\end{array}$ & $\begin{array}{c}\text { PSNR } \\
(\mathrm{dB})\end{array}$ & $\begin{array}{c}\text { Time } \\
(\mathrm{sec})\end{array}$ & $\begin{array}{c}\text { Sparcity } \\
(\%)\end{array}$ & $\begin{array}{c}\text { PSNR } \\
(\mathrm{dB})\end{array}$ & $\begin{array}{c}\text { Time } \\
(\mathrm{sec})\end{array}$ & $\begin{array}{c}\text { Sparcity } \\
(\%)\end{array}$ \\
\hline $\begin{array}{l}\text { Gaussian } \\
\text { Random } \\
\text { Matrix }\end{array}$ & 20.24 & 7.08 & 36.30 & 28.52 & 1.80 & 50.07 & 28.11 & 1.95 & 50.03 \\
\hline $\begin{array}{l}\text { Bernoulli } \\
\text { Random } \\
\text { Matrix }\end{array}$ & 17.76 & 3.75 & 97.89 & 25.39 & 1.28 & 66.46 & 25.36 & 1.19 & 49.73 \\
\hline $\begin{array}{l}\text { Laplace } \\
\text { Random } \\
\text { Matrix }\end{array}$ & 20.20 & 7.30 & 68.22 & 28.72 & 1.65 & 54.27 & 28.79 & 1.57 & 49.00 \\
\hline $\begin{array}{l}\text { Logistic } \\
\text { Random } \\
\text { Matrix }\end{array}$ & 20.28 & 6.45 & 50.54 & 28.47 & 1.26 & 53.14 & 28.92 & 1.31 & 43.77 \\
\hline $\begin{array}{l}\text { Cauchy } \\
\text { Random } \\
\text { Matrix }\end{array}$ & 19.95 & 8.82 & 60.62 & 28.17 & 1.83 & 46.33 & 28.54 & 1.86 & 46.45 \\
\hline
\end{tabular}

matrix is able to achieve performance closer to the maximum one in terms of both metrics. Thus, SDPC-PlusSQ method has attained better PSNR value, sparsity and computation time than other two predictive coding methods. It is evident that, DPCM-Plus-SQ is the second in performance and better than SQ method.

For the predictive coding approaches, the compressive sensing results attained based on different measurement matrices for chest CT dataset is presented in Table 2. For SQ method, the Gaussian random matrix has yielded maximum PSNR value $(20.28 \mathrm{~dB})$ and minimum computation time $(7.08 \mathrm{sec}$.).For the same, Bernoulli random matrix has shown least performance in terms of PSNR and sparsity (97.89\%), Logistic random matrices needs more computation time. However, Bernoulli and Logistic matrices show least performance in terms of computation time, PSNR value and sparsity, respectively. For DPCM-Plus-SQ method, maximum PSNR value $(28.72 \mathrm{~dB})$, computation time $(1.28 \mathrm{sec}$.) and sparsity $(54.27 \%)$ are attained by Laplace random matrix. Bernoulli random matrix has shown least performance in terms of PSNR and sparsity (97.89\%), Cauchy random matrix needs more computation time. For SDPC-Plus-SQ method, maximum PSNR value (28.92) is attained for Logistic random matrix with less computation time (1.31sec.) and sparsity (43.77\%). The least performance is for Gaussian and Bernoulli random matrices in terms of PSNR value and computation time, respectively. On the whole, Logistic random matrix is able to achieve performance closer to the maximum one in terms of both metrics. Comparatively, SDPC-Plus-SQ method has attained maximum PSNR value, least computation time and sparsity, thus better than other two predictive coding methods. It is evident that, DPCM-Plus-SQ is the second in performance and better than SQ method.

The compressive sensing results obtained for predictive approaches using different measurement matrices experimenting with shoulder MRI dataset are given in Table 3. For SQ method, the Bernoulli random matrix has yielded maximum PSNR value $(26.74 \mathrm{~dB})$, minimum computation time $(5.66 \mathrm{sec})$ and sparsity $(99.66 \%)$. whereas Cauchy random matrix requires more computation time. For DPCM-Plus-SQ method, maximum PSNR value $(40.74 \mathrm{~dB})$ least computation time $(4.59 \mathrm{sec}$.) and sparsity $(65.79 \%)$ is attained by Gaussian random matrix. However, Gaussian and Bernoulli matrices show least performance in terms of computation time and PSNR value, respectively. For SDPC-Plus-SQ method, maximum PSNR value ( $40.75 \mathrm{~dB}$ ), least computation time (5.45sec.) and sparsity (54.93\%) are attained for Laplace random matrix and Bernoulli random matrix 


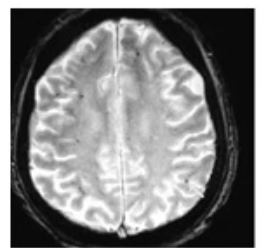

Original image

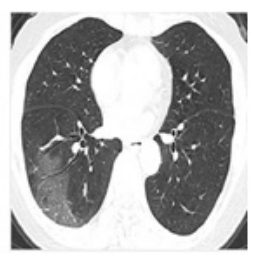

Original image



Original image



Bernoulli and SQ

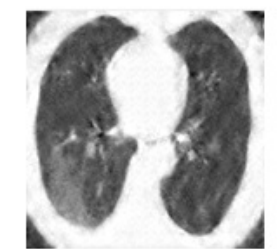

Bernoulli and SQ



Cauchy and SQ

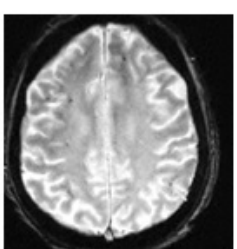

Laplace and SDPC

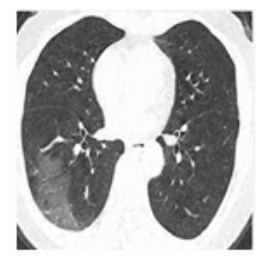

Laplace and SDPC



Laplace and SDPC

Figure 4: Reconsructed images using measurement matrices (a) Original image (b) Least performance of reconstructed images (c) Best performance of reconstructed images

needs less computation time. The least performance is for Bernoulli and Cauchy random matrices in terms of PSNR value and computation time, respectively. On the whole, Laplace random matrix is able to achieve performance closer to the maximum one in terms of both metrics. Comparatively, SDPC-Plus-SQ method has attained maximum PSNR value least computation time and sparsity, thus better than other two predictive coding methods. It is evident that, DPCM-Plus-SQ is the second in performance and better than SQ method.

In Fig.4, the reconstructed images for the sample brain MRI, chest CT and shoulder MRI images with reference to different measurement matrices applied in the predictive coding approaches are presented. For brain MRI images, the SQ method using Bernoulli random matrix has suffered with maximum level of noise and low intensity contrast indicated by $99 \%$ of sparsity. On the other hand, the SDPC method using Laplace random matrix has yielded an enhanced image with $49 \%$ of sparsity. For chest CT images, the SDPC method using Logistic random matrix works well with $43 \%$ of sparsity and worst by Bernoulli random matrix with $97 \%$ of sparsity. For shoulder MRI images, the Laplace random matrix has yielded better enhanced image with $57 \%$ of sparsity. For the same, Cauchy random matrix output shows low intensity contrast image with $65 \%$ of sparsity. On the whole, Laplace random matrix is able to reconstruct the image well, which is indicted with sparsity $43 \%$. From the results it is understood that the maximum level sparsity relates to highly noisy image and the sparsity level attained below $60 \%$ for SDPC method is a significant one. Overall the SDPCPlus-SQ method using Laplace random matrix has attained better reconstructed image with minimum sparsity, thus better than other two predictive coding methods. It is evident that, DPCM-Plus-SQ is the second in performance of reconstructed images and better than SQ method. In this paragraph, the Laplace distribution based sampling which is the underline for better performance by Laplace random matrix towards compressive sensing is presented. The Laplace distribution, also called the double exponential and its probability function is similar to the normal distribution. Its main characteristic is the way it models the probability of deviations from a central value, also known as errors. 
Table 3: Performance Evaluation of Shoulder-MRI

\begin{tabular}{|l|c|c|c|c|c|c|c|c|c|}
\hline $\begin{array}{l}\text { Measurement } \\
\text { Matrix Distribution }\end{array}$ & \multicolumn{3}{|c|}{ SQ } & \multicolumn{3}{c|}{ DPCM-Plus-SQ } & \multicolumn{3}{c|}{ SDPC-Plus-SQ } \\
\cline { 2 - 10 } & $\begin{array}{c}\text { PSNR } \\
(\mathrm{dB})\end{array}$ & $\begin{array}{c}\text { Time } \\
(\mathrm{sec})\end{array}$ & $\begin{array}{c}\text { Sparcity } \\
(\%)\end{array}$ & $\begin{array}{c}\text { PSNR } \\
(\mathrm{dB})\end{array}$ & $\begin{array}{c}\text { Time } \\
(\mathrm{sec})\end{array}$ & $\begin{array}{c}\text { Sparcity } \\
(\%)\end{array}$ & $\begin{array}{c}\text { PSNR } \\
(\mathrm{dB})\end{array}$ & $\begin{array}{c}\text { Time } \\
(\mathrm{sec})\end{array}$ & $\begin{array}{c}\text { Sparcity } \\
(\%)\end{array}$ \\
\hline $\begin{array}{l}\text { Gaussian } \\
\text { Random } \\
\text { Matrix }\end{array}$ & 21.76 & 5.66 & 79.68 & 40.74 & 4.59 & 65.79 & 40.62 & 4.79 & 49.83 \\
\hline $\begin{array}{l}\text { Bernoulli } \\
\text { Random } \\
\text { Matrix }\end{array}$ & 26.74 & 2.57 & 99.66 & 38.12 & 2.51 & 89.17 & 37.77 & 2.31 & 54.78 \\
\hline $\begin{array}{l}\text { Laplace } \\
\text { Random } \\
\text { Matrix }\end{array}$ & 21.88 & 4.97 & 84.53 & 40.49 & 4.06 & 60.43 & 40.75 & 5.45 & 57.93 \\
\hline $\begin{array}{l}\text { Logistic } \\
\text { Random } \\
\text { Matrix }\end{array}$ & 23.14 & 7.97 & 77.50 & 40.27 & 4.28 & 65.80 & 40.42 & 4.05 & 54.59 \\
\hline $\begin{array}{l}\text { Cauchy } \\
\text { Random } \\
\text { Matrix }\end{array}$ & 21.08 & 7.22 & 65.89 & 40.69 & 6.73 & 62.09 & 40.42 & 5.22 & 53.40 \\
\hline
\end{tabular}

The Laplace (or double exponential) distribution, like the normal, has a distinguished history in statistics. In the literature, the Gaussian distribution achieves the improved output in compressive sensing for the same, the Laplace distribution has similar to Gaussian distribution. Hence, the Laplace distribution performs well on measurement matrix for reconstructed medical images. It has applications in image and speech recognition, ocean engineering, hydrology, and finance. Indeed, there has been a surge of interest towards employing Laplace distribution for generating random numbers among the research community.

\section{Conclusion}

In this paper, various measurement matrices with directional predictive coding have been experimented towards compressive sensing of three types of medical images. The Laplace random matrix that satisfies the RIP condition, gives the best image reconstruction results. The performance of the measurement matrices are evaluated based on the experimentation using Shoulder MRI, Chest radiograph and Brain MRI images. The SDPC method using Laplace matrix has shown the maximum performance in terms of PSNR, computation time and sparsity. The improvement achieved towards compressive sensing of medical images in terms of sparsity, PSNR and running time is an indication in serving the purpose of transmission, storage and reconstruction of large medical images of high quality with lesser bandwidth, memory and quality reconstruction, respectively. This in turn can help in diagnosing diseases too. Thus, this research outcome would be greatly beneficial to medical authorities in the above perspective. In future work, the compressive sensing approach considered in this work can be tested with the recently developed restricted structural random matrix. Also, it can be extended to more types of medical images. 


\section{References}

[1] Arjoune, Youness, et al. "A performance comparison of measurement matrices in compressive sensing." International Journal of Communication Systems 31.10 (2018): e3576.

[2] Bai, Huihui, Anhong Wang, and Mengmeng Zhang. "Compressive sensing for DCT image." In 2010 International Conference on Computational Aspects of Social Networks, pp. 378-381. IEEE, 2010.

[3] Candes, E.J., Tao, T., "Decoding by linear programming". IEEE Trans. Inform. Theory 51(2005), no.12, 42034215.

[4] Chen, C., Tramel, E. W., Fowler, J. E. (2011, November).Compressed-sensing recovery of images and video using multihypothesis predictions. In 2011 conference record of the forty fifth asilomar conference on signals, systems and computers (ASILOMAR) (pp. 1193-1198). IEEE.

[5] Chen, Scott Shaobing, David L. Donoho, and Michael A. Saunders. "Atomic decomposition by basis pursuit." SIAM review 43, no. 1 (2001): 129-159.

[6] Donoho, D. L. "Compressed sensing: IEEE Transactions on Information Theory, 52, no. 4, 12891306." (2006).

[7] Duarte, Marco F., et al. "Single-pixel imaging via compressive sampling." IEEE signal processing magazine 25.2 (2008): 83-91.

[8] Gan, L. (2007, July). Block compressed sensing of natural images. In 2007 15th International conference on digital signal processing (pp. 403-406).

[9] Mun, S., Fowler, J. E. (2009, November). Block compressed sensing of images using directional transforms. In 2009 16th IEEE international conference on image processing (ICIP) (pp. 3021-3024).

[10] Mun, S., Fowler, J. E. (2012, August). DPCM for quantized block-based compressed sensing of images. In 2012 Proceedings of the 20th European Signal Processing Conference (EUSIPCO) (pp. 1424-1428).

[11] Rani, Meenu, S. B. Dhok, and R. B. Deshmukh. "A systematic review of compressive sensing: Concepts, implementations and applications.” IEEE Access 6 (2018): 4875-4894.

[12] Xiao, Ying, et al. "Compressed sensing based apple image measurement matrix selection." International Journal of Distributed Sensor Networks 11.7 (2015): 901073.

[13] Yu, Yao, Athina P. Petropulu, and H. Vincent Poor. "Measurement matrix design for Compressive sensing based MIMO radar.” IEEE Transactions on Signal Processing 59.11 (2011): 5338-5352.

[14] Zhang, Z., Xu, Y., Yang, J., Li, X., Zhang, D. (2015). A survey of sparse representation: algorithms and applications. IEEE access, 3, 490-530.

[15] G.Kowsalya, H.A.Christianl, D.A.chandy, C.Bajaj. "Analysis of the impact of measurement matrices in compressive sensing for medical images", Advances in Mathematics: Scientific Journal, 2020.

[16] Zhang, J., Zhao, D., Jiang, F. (2013, September). Spatially directional predictive coding for block-based compressive sensing of natural images. In 2013 IEEE International Conference on Image Processing (pp. 1021-1025).

[17] Selvan, S. E., Subathra, M. S. P., Christinal, A. H., Amato, U. (2013). On the benefits of Laplace samples in solving a rare event problem using cross-entropy method. Applied Mathematics and Computation, 225, 843-859. 
[18] Bajaj, C., Goswami, S. (2009). Modeling cardiovascular anatomy from patient-specific imaging. In Advances in computational vision and medical image processing (pp. 1-28). Springer, Dordrecht.

[19] Alex, D. M., Chandy, D. A. (2020, July). Evaluation of Inpainting in Speckled and Despeckled 2D Ultrasound Medical Images. In 2020 Advanced Computing and Communication Technologies for High Performance Applications (ACCTHPA) (pp. 221-225). IEEE.

[20] Carnero, J., Christinal, H. A., Daniel, D., Reina-Molina, R., Subathra, M. S. P. (2014). Improved parallelization of an image segmentation bio-inspired algorithm. In Proceedings of the Second International Conference on Soft Computing for Problem Solving (SocProS 2012), December 28-30, 2012 (pp. 75-82). Springer, New Delhi.

[21] Sun, T., Cao, H., Blondel, P., Guo, Y., Shentu, H. (2018). Construction of Measurement Matrix Based on Cyclic Direct Product and QR Decomposition for Sensing and Reconstruction of Underwater Echo. Applied Sciences, 8(12), 2510.

[22] Zhang, Z., Xu, Y., Yang, J., Li, X., Zhang, D. (2015). A survey of sparse representation: algorithms and applications. IEEE access, 3, 490-530.

[23] Kong, W., Agarwal, P. P. (2020). Chest imaging appearance of COVID-19 infection. Radiology: Cardiothoracic Imaging, 2(1), e200028.

[24] Gulko, E., Oleksk, M. L., Gomes, W., Ali, S., Mehta, H., Overby, P., ... Rozenshtein, A. (2020). MRI brain findings in 126 patients with COVID-19: initial observations from a descriptive literature review. American Journal of Neuroradiology, 41(12), 2199-2203. 